\title{
MusculoskeletalDisordersandTreatment
}

\section{Spontaneous Proximal Tibiofibular Joint Ganglion Cyst as a Rare Cause of Acute Foot Drop: A Case Report}

\section{Nirguna Thalla, MD, $M B A^{1,2,3}$, W Daniel White, $M D^{1,2,3}$, J Taylor Mansfield, $D O^{1,2,3}$, Jason $M$ Souza, $M D^{3}$ and Matthew $E$ Miller, $M D^{3}$}

\author{
${ }^{1}$ Department of Rehabilitation Medicine, MedStar Georgetown University, Washington, DC, USA \\ ${ }^{2}$ Physical Medicine and Rehabilitation, MedStar National Rehabilitation Hospital, Washington, DC, USA \\ ${ }^{3}$ Department of Physical Medicine and Rehabilitation, Walter Reed National Military Medical Center, \\ Bethesda, MD, USA
}

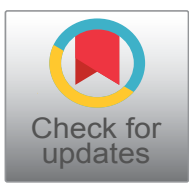

*Corresponding author: Nirguna Thalla, MD, MBA, Physical Medicine and Rehabilitation, MedStar National Rehabilitation Hospital, 102 Irving St. NW, Washington, DC 20010, USA, Tel: 248-497-5997

\begin{abstract}
Fibular neuropathy has several well understood causes. An extraneural ganglion cyst is a poorly understood cause of this clinically important neuromuscular condition. We report a case of a 50-year-old male who developed acute foot drop caused by a compressive ganglion cyst infiltrating the common fibular nerve at the proximal tibiofibular joint. Intraneural ganglion cysts or synovial cysts development affecting the deep fibular nerve has been reported in the literature. To our knowledge, an extraneural ganglion cyst is an extremely rare cause of lower extremity nerve palsy. In this article, we review the case details, explore the incidence, pathophysiology, the detailed workup and discuss the clinical importance and management of this phenomenon.
\end{abstract}

\section{Keywords}

Foot drop, Ganglion cyst, Tibiofibular joint, Fibular nerve, Electrodiagnostic study

\section{Abbreviations}

AFO: Ankle Foot Orthosis; EDB: Extensor Digitorum Brevis; EDL: Extensor Digitorum Longus, EDX: Electrodiagnostic Study; EHL: Extensor Hallucis Longus; EMG: Electromyography; MRI: Magnetic Resonance Imaging; PTFJ: Proximal Tibiofibular Joint; TA: Tibialis Anterior; US: Ultrasound

\section{Introduction}

Foot drop is the inability to lift the forefoot due to weakness or paralysis of the foot dorsiflexors. Foot drop is a debilitating condition resulting in impaired ambulation and increases the risk for falls and subsequent injuries. Compressive disorders resulting in chronic nerve damage to the Common/Deep fibular nerves are a well-recognized source for foot drop. These can include weight loss, prolonged bedridden status, tight casts, lumbar radiculopathy and bone metastasis involving the fibular head. Acute causes of foot drop tend to be related to traumatic injuries commonly associated with orthopedic injuries [1]. Infiltrating, space occupying lesions such as ganglion cysts are one subtype that are classified as benign soft tissue tumors and are most commonly encountered in the wrist. They are frequently asymptomatic [2]. Lower extremity ganglion cysts are less common such as a proximal tibiofibular joint (PTFJ) ganglion cyst which was first described in 1891 [3]. PTFJ ganglion cysts have since been a rarely occurring phenomenon. Our case looks at a rare disease presenting uncommonly resulting in a widely recognized condition. We describe a patient with a spontaneous PTFJ ganglion cyst resulting in acute foot drop.

\section{Case Description}

This patient is a 50-year-old male with no significant past medical history who developed acute onset right ankle pain while driving. Shortly thereafter, lateral knee pain developed. Over the next several hours to days the pain persisted while he also developed ankle dorsiflexion weakness. The patient was evaluated in

Citation: Thalla N, White WD, Mansfield JT, Souza JM, Miller ME (2021) Spontaneous Proximal Tibiofibular Joint Ganglion Cyst as a Rare Cause of Acute Foot Drop: A Case Report. J Musculoskelet Disord Treat 7:095. doi.org/10.23937/2572-3243.1510095 Accepted: May 08, 2021: Published: May 10, 2021

Copyright: (c) 2021 Thalla N, et al. This is an open-access article distributed under the terms of the Creative Commons Attribution License, which permits unrestricted use, distribution, and reproduction in any medium, provided the original author and source are credited. 
Table 1: Initial motor nerve conduction study of the right lower extremity TA and EDB.

Motor Nerve Conduction:

\begin{tabular}{|c|c|c|c|c|c|c|}
\hline Nerve and Site & $\begin{array}{l}\text { Lat } \\
\text { ms }\end{array}$ & $\begin{array}{l}\text { Amp } \\
m V\end{array}$ & Segment & $\begin{array}{l}\text { Dist } \\
\mathrm{mm}\end{array}$ & $\begin{array}{l}\text { Lat Diff } \\
\text { ms }\end{array}$ & $\begin{array}{l}\text { CV } \\
\mathrm{m} / \mathrm{s}\end{array}$ \\
\hline \multicolumn{5}{|c|}{ Peroneal.R to Extensor digitorum brevis. $R$} & & \\
\hline EDB Ankle & & & Extensor digitorum brevis-EDB Ankle & 80 & & \\
\hline Fib Head & NR & NR & EDB Ankle-Fib Head & 330 & & \\
\hline Popliteal Fossa & NR & NR & Fib Head-Popliteal Fossa & 110 & & \\
\hline \multicolumn{7}{|c|}{ Peroneal. $\mathbf{R}$ to Tibialis anterior. $R$} \\
\hline Fib Head & 6.7 & 0.9 & & & & \\
\hline \multirow[t]{2}{*}{ Popliteal Fossa } & 8.5 & 0.4 & Tibialis anterior-Fib Head & 330 & 6.7 & \\
\hline & & & Fib Head-Popliteal Fossa & 110 & 1.8 & 61 \\
\hline
\end{tabular}

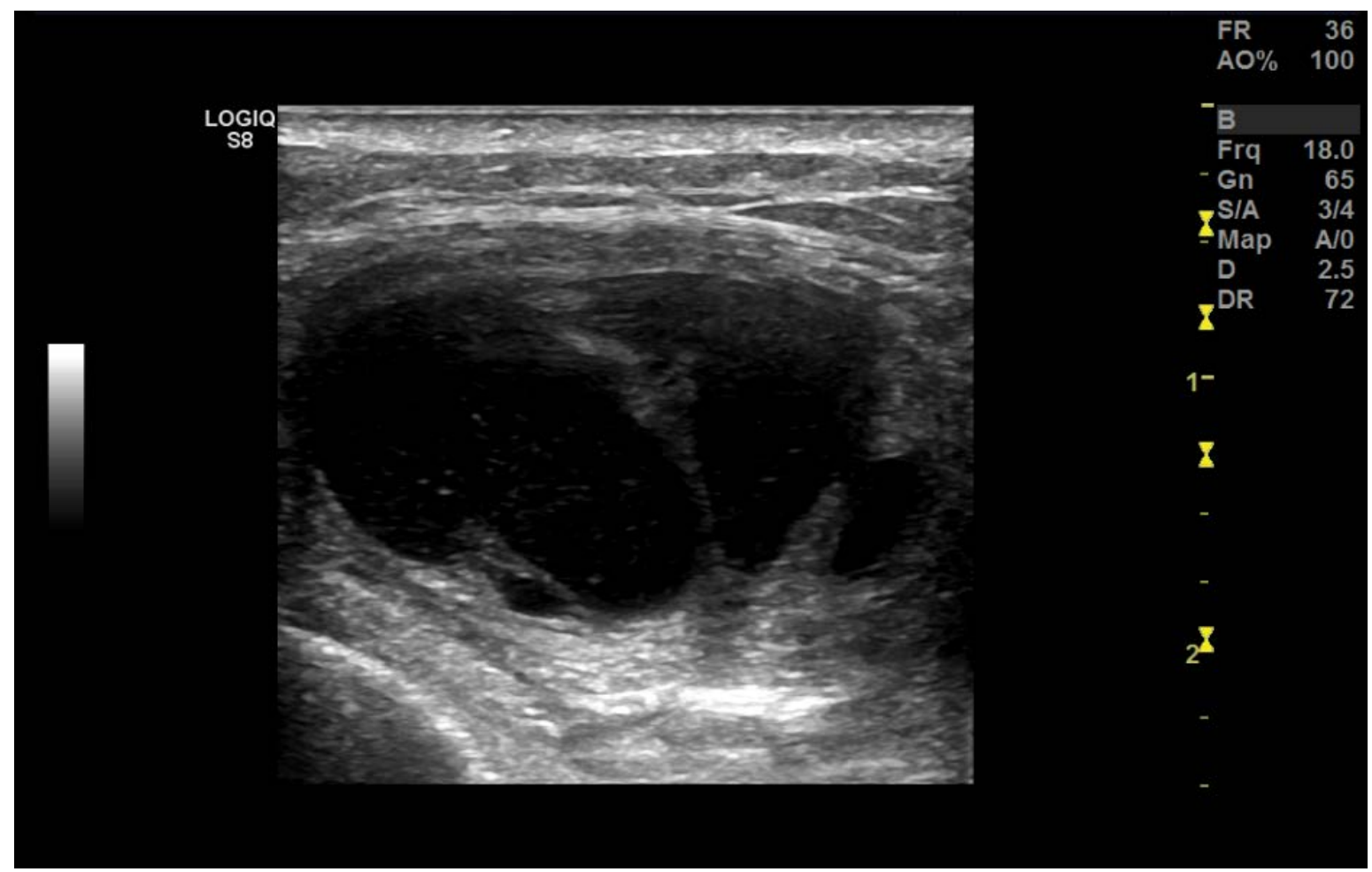

Figure 1: Ultrasound imaging depicting a cyst in the tibiofibular joint space. The deep fibular nerve is in the 5 o'clock position in this photograph (deep to the cyst). The fibular bone is to the left and the right of the image is anteromedial.

the emergency department 4 days after symptom onset with persistent pain/weakness. X-Rays of the ankle and tibia/fibula were unremarkable. He was referred to neurology. Neurology recommended an electrodiagnostic (EDX) study, ankle foot orthosis (AFO) and physical therapy. The EDX study was performed 4 weeks after symptom onset, at which time ankle dorsiflexion/toe extension strength was $0 / 5$ and numbness was reported in the anterolateral leg and dorsal foot. Otherwise, strength testing was unremarkable in other muscle groups and he denied any ongoing pain. The EDX study was remarkable for severe motor axonal deep fibular neuropathy without axonal continuity to the extensor digitorum brevis (EDB) but preserved axonal continuity to the tibialis anterior (TA). Otherwise, there was no EDX evidence of superficial fibular neuropathy, tibial neuropathy, or lumbar radiculopathy. The results of the EDX study are included in Table 1. An ultrasound was performed immediately following the EDX study which demonstrated a loculated collection anterior to the fibular neck with extension to the PTFJ (Figure 1). Subsequent MRI confirmed a PTFJ ganglion cyst resulting in deep fibular nerve compression against the proximal fibula (Figure 2).

Surgical cyst excision was performed 10 weeks after symptom onset. Figure 3 depicts the ganglion cyst that was discovered intraoperatively, and ultimately excised. At 3 months post-operation, the patient appreciated pain resolution, sensation abnormality limited to the medial malleolus and distally over the dorsum of the foot. Strength of toe extension/dorsiflexion was noted to be antigravity with 10 degrees extension. Additional- 


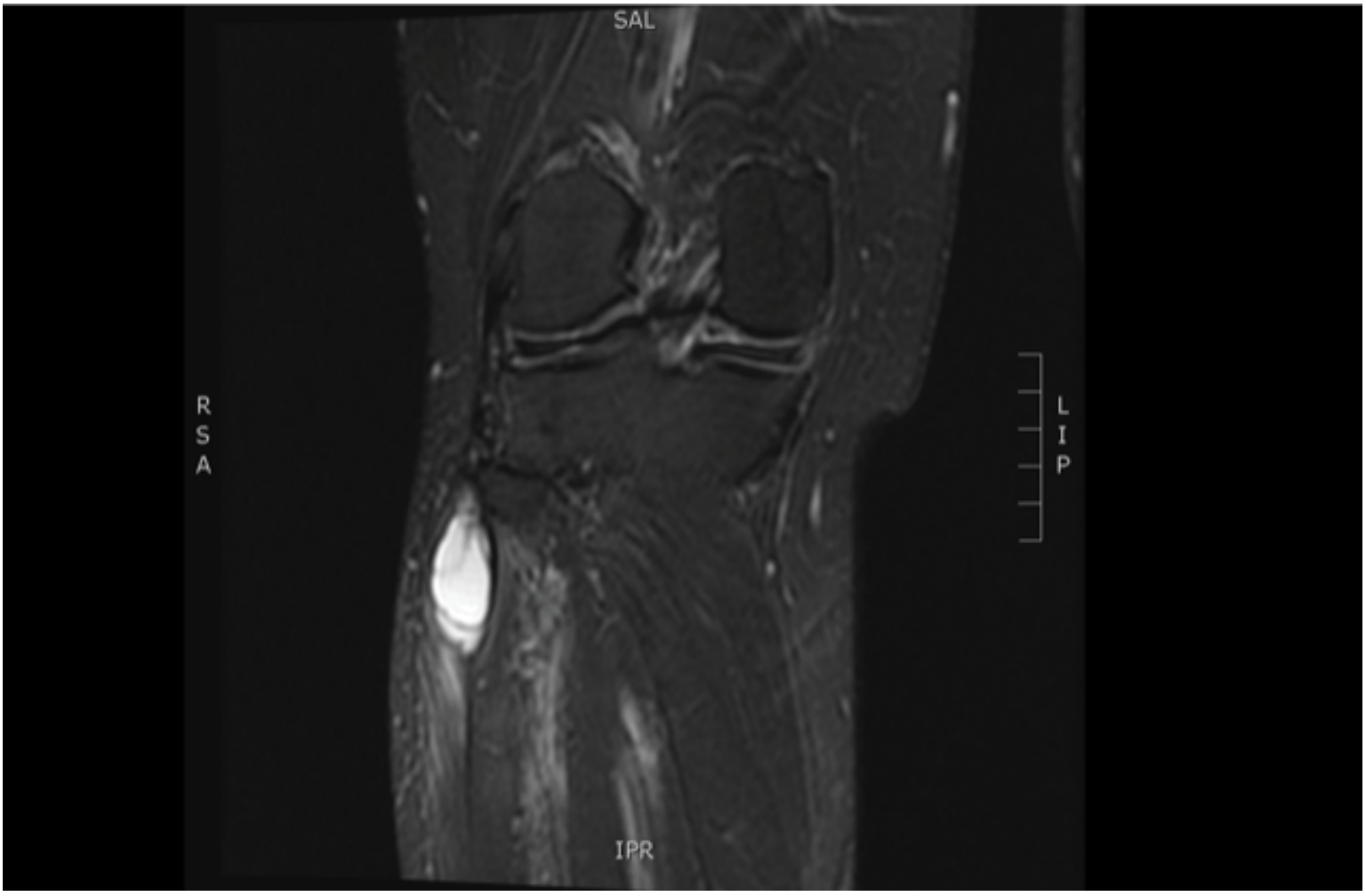

Figure 2: MRI (Coronal T2 fat saturated) of the right knee and proximal lower leg. Ganglion cyst arising from the tibiofibular joint is present in the proximal lateral compartment of the leg. The mass measures approximately $4.2 \times 2.1 \times 1.8 \mathrm{~cm}$ and demonstrates multiple T2 hyperintense loculated cystic foci.

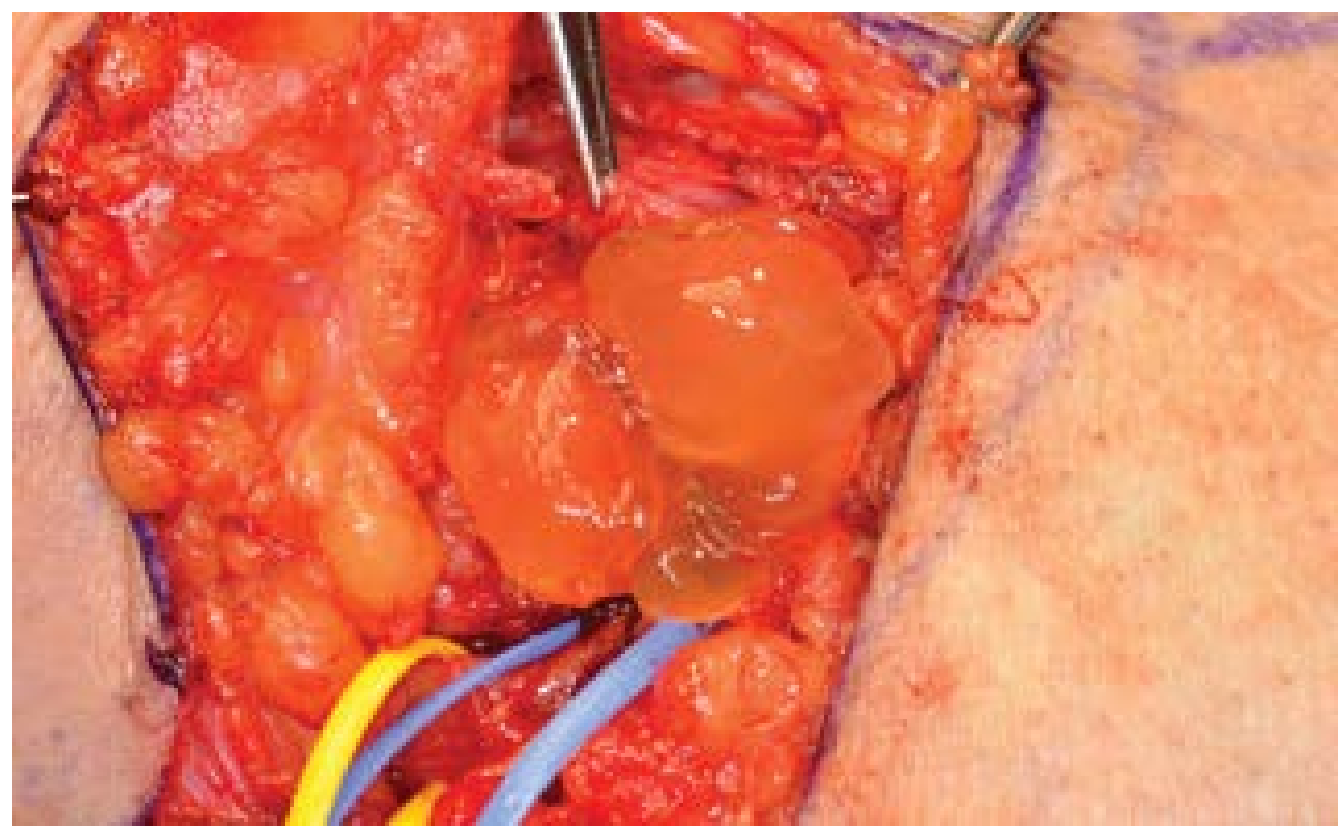

Figure 3: Intraoperative specimen. Pathology report: Right Peroneal nerve articular branch and cyst. It consists of both a 0.5 $\times 0.5 \times 0.2 \mathrm{~cm}$ irregular soft tissue fragment and a separate $0.5 \times 0.5 \times 0.5$ pink-tan, soft tubular tissue fragment.

ly, there was no ongoing evidence of impaired toe clearance or compensatory gait pattern.

\section{Discussion}

The PTFJ consists of the articulation between the fibular head and the lateral condyle of the tibia. This surface is lined with a synovial membrane and is surrounded by an articular capsule. The common fibular nerve arises as a division of the sciatic nerve. The sciatic nerve divides into its fibular and tibial components proximal to the popliteal fossa. Here the common fibular nerve continues to dive laterally and approaches the anterolateral region of the leg by arching around the posterior aspect of the fibular head. At the level of the fibular neck, the nerve traverses posterior to anterior within $25 \mathrm{~mm}$ from the actual tip of the fibular neck [4]. This creates a very 
proximal distance to the PTFJ and any pathological findings within that joint space.

The pathogenesis of PTFJ ganglion cysts remains uncertain. The cyst itself consists of a gelatinous material containing mainly hyaluronic acid and is thicker than nearby synovial fluid [2]. The literature has pointed to a few theories that can offer an explanation. The idea that the cyst is a simple herniation of the joint capsule has largely been debunked due to a lack of synovial lining [5]. The articular theory originating in 1926 is a leading concept, suggesting that ganglion cysts result from mucinous degeneration of connective tissue secondary to chronic damage [6]. The subsequent impact to the nerve is the result of degenerated joint fluid passing through a capsular defect into an articular branch of the fibular nerve that results in dissection [6].

Given the rarity of PTFJ ganglion cysts, we performed a literature search to identify the number of cases reported that show involvement of the fibular nerve. We were able to identify several cases, however, a majority of these cases involved intraneural ganglion cysts or synovial cysts. Given a discrepancy in the reporting of cyst characterization, we were unable to identify a specific incidence of extraneural ganglion PTFJ cysts resulting in deep fibular nerve palsy. Additionally, we reviewed an observational cross-sectional study that investigated the prevalence of PTFJ cysts in all MRI images of the knee in an outpatient center from 2000-2001 [7]. A total of 654 knee MRI scans were performed. This patient population consisted of an average age of 43.4 years who complained of knee pain. The most common clinical diagnosis in these 654 patients was meniscal tear (42.8\%). Five patients had a PTFJ ganglion cyst detected on MRI, yielding a low prevalence of $0.76 \%$ consistent with our understanding of PTFJ ganglion cysts [7].

Fibular nerve palsy has a typical presentation warranting its suspicion. It presents as spontaneous pain localized to the fibular neck that may then refer downward. Thereafter, development of muscle weakness of the TA, EDB becomes the most common concern [8]. For patients presenting with this clinical pattern, there are several diagnostic steps to be considered. Initial X-rays should be performed to rule out acute fractures that may result in nerve injury. If clinical weakness is present, the clinician should use EDX studies to localize and evaluate the degree of nerve injury. The ability to use point-of-care ultrasound following the EDX studies to diagnose PTFJ cyst is a novel approach that was used in this case. US evaluation allows for direct visualization of the cyst and may show edema, thickening, and loss of continuity of the nerve fibers [9]. This approach allows for a shortened time to diagnosis and treatment. Prolonged nerve damage without appropriate treatment will affect the recovery potential, especially in cases that require surgical intervention. Following initial diagnosis using US, MRI is used to further characterize the lesion.
Prognosis tends to be good regarding compressive etiologies of fibular nerve injuries. One retrospective study followed the natural history of 25 patients with non-traumatic compressive fibular mononeuropathy. $90 \%$ of patients experienced clinically significant recovery of weakness with better outcomes reported in those with NCS/EMG findings indicative of demyelination rather than axonal loss. However, significant recovery was seen in axonal lesions as well [10]. Additionally, better outcomes were observed in younger patients. Surgical vs. conservative treatment of a compressive etiology for deep fibular mononeuropathy is an important consideration. Conservative treatment can lead to a 1-2 year rehabilitative process requiring long term use of a brace. Surgical decompression accelerates recovery and has been shown to be more effective in relieving the conduction abnormality [4].

In our case, the surgical approach was pursued, and the cyst was excised with no complications. The patient returned for a progress evaluation approximately 3 months after the excision. He reported a significant improvement in quality of life due to pain resolution and improved functionality of his muscles. A follow up EDX study was performed which revealed no axonal continuity to the EDB and Extensor Hallucis Longus (EHL). The detailed results of this EDX study are reported in Table 2. The toe extension that was observed is attributed to reinnervation of the Extensor Digitorum Longus (EDL). Injuries to peripheral nerves can be difficult to grade but a majority fall under the class of Neuropraxia. However, in our case, axonotmesis appears to be the primary mechanism of injury. Axonotmesis is a more serious injury than neuropraxia and is defined as injury resulting in disruption of the axon and myelin sheath with preservation of the neuronal connective tissue. Wallerian Degeneration results due to loss of axoplasmic flow [11]. Based on the Seddon and Sunderland classification of Nerve Injury, our patient's injury is consistent with a Grade 2 classification [12]. The recovery process is expected to be delayed and progress over months. He will require ongoing follow up, especially given the possibility of cyst recurrence that may require proximal tibiofibular joint arthrodesis for definitive treatment [13].

\section{Conclusion}

PTFJ ganglion cysts are rare entities that can cause nerve entrapment to the fibular nerve or its branches. Fibular nerve palsy is a clinically disturbing phenomenon that is associated with significant impairment to daily living functions. This case illustrates the importance of a systematic diagnostic approach to unveiling a rare, poorly understood disease and careful management to continue to facilitate functional recovery in this patient with the optimistic outlook for preventing long term morbidity.

\section{Author Disclosures}

None of the authors has any conflict of interest to disclose. 
Table 2: Follow up ( 3 months post-operation): Motor Nerve Conduction study and Needle EMG of the Right lower extremity TA and EDB.

\section{Motor Nerve Conduction:}

\begin{tabular}{|c|c|c|c|c|c|c|}
\hline Nerve and Site & $\begin{array}{l}\text { Lat } \\
\mathrm{ms}\end{array}$ & $\begin{array}{l}\text { Amp } \\
m V\end{array}$ & Segment & $\begin{array}{l}\text { Dist } \\
\mathrm{mm}\end{array}$ & $\begin{array}{l}\text { Lat Diff } \\
\mathrm{ms}\end{array}$ & $\begin{array}{l}\text { CV } \\
\mathrm{m} / \mathrm{s}\end{array}$ \\
\hline \multicolumn{5}{|c|}{ Peroneal.R to Extensor digitorum brevis.R } & & \\
\hline EDB Ankle & NR & NR & Extensor digitorum brevis-EDB Ankle & 80 & & \\
\hline Fib Head & NR & NR & EDB Ankle-Fib Head & & & \\
\hline Popliteal Fossa & 18.5 & 0.2 & Fib Head-Popliteal Fossa & & & NR \\
\hline \multicolumn{4}{|c|}{ Peroneal.R to Tibialis anterior.R } & & & \\
\hline Fib Head & 3.5 & 0.6 & & & & \\
\hline \multirow[t]{2}{*}{ Popliteal Fossa } & 4.8 & 2.1 & Tibialis anterior-Fib Head & & 3.5 & \\
\hline & & & Fib Head-Popliteal Fossa & & 1.3 & \\
\hline
\end{tabular}

Needle EMG Examination:

\begin{tabular}{|c|c|c|c|c|c|c|c|c|c|c|c|}
\hline \multirow[t]{2}{*}{ Muscle } & \multirow{2}{*}{$\begin{array}{l}\text { Insertion } \\
\text { Activity }\end{array}$} & \multicolumn{4}{|c|}{ Spontaneous Activity } & \multicolumn{5}{|c|}{ Volitional MUAPs } & \multirow[b]{2}{*}{ Comment } \\
\hline & & Fibs & PSW & Fasc & Other & Effort & Recruit & Dur & Amp & Poly & \\
\hline Tibialis anterio. $\mathrm{R}$ & Normal & 0 & 0 & None & & Normal & Normal & SI Incr & SI Incr & Rare & \\
\hline $\begin{array}{l}\text { Extensor hallucis } \\
\text { longus. } R\end{array}$ & Increased & $2+$ & $2+$ & None & & Normal & & Normal & Normal & None & \\
\hline
\end{tabular}

\section{Funding Received}

None.

\section{Author's Contribution}

All authors contributed to literature review, data collection, interpretation and writing of the manuscript.

"We confirm that we have read the Journal's position on issues involved in ethical publication and affirm that this report is consistent with those guidelines".

\section{References}

1. Nori SL, Stretanski MF (2020) Foot drop. In: StatPearls. StatPearls Publishing, Treasure Island (FL).

2. Gude W, Morelli V (2008) Ganglion cysts of the wrist: Pathophysiology, clinical picture, and management. Curr Rev Musculoskelet Med 1: 205-211.

3. Aymen $F$, Jacem $S$, Youssef $O$, Issam $A$, Abderrazek $A$ (2019) Peroneal nerve palsy caused by a synovial cyst of the proximal tibiofibular joint: A report of two cases and review of the literature. Pan Afr Med J 34: 115.

4. Schrijver FD, Simon JP, Smet LD, Fabry G (1998) Ganglia of the superior tibiofibular joint: Report of three cases and review of the literature. Acta Orthop Belg 64: 233-241.

5. Neto N, Nunnes P (2016) Spectrum of MRI features of ganglion and synovial cysts. Insights Imaging 7: 179-186.
6. Gregush RE, Habusta SF (2020) Ganglion Cyst. In: StatPearls. StatPearls Publishing, Treasure Island (FL).

7. Ilahi OA, Younas SA, Labbe MR, Edson SB (2003) Prevalence of ganglion cysts originating from the proximal tibiofibular joint: A magnetic resonance imaging study. Arthroscopy 19: 150-153.

8. Alsahhaf A, Renno W (2016) Ganglion cyst at the proximal tibiofibular joint in a patient with painless foot drop. Pain Physician 19: E1147-E1160.

9. Bendszus M, Stoll G (2005) Technology insight: Visualizing peripheral nerve injury using MRI. Nat Clin Pract Neurol 1: 45-53.

10. Bsteh G, Wanschitz JV, Gruber H, Seppi K, Löscher WN (2013) Prognosis and prognostic factors in non-traumatic acute-onset compressive mononeuropathies--radial and peroneal mononeuropathies. Eur J Neurol 20: 981-985.

11. Faubel C (2010) Nerve injury classifications - Seddon's and Sunderland's.

12. Menorca RMG, Fussell TS, Elfar JC (2013) Nerve physiology: Mechanisms of injury and recovery. Hand Clin 29: 317330.

13. Miskovsky S, Kaeding C, Weis L (2004) Proximal tibiofibular joint ganglion cysts: Excision, recurrence, and joint arthrodesis. Am J Sports Med 32: 1022-1028. 